\title{
DNMT family induces down-regulation of NDRG1 via DNA methylation and clinicopathological significance in gastric cancer
}

\author{
Xiaojing Chang ${ }^{\text {Equal first author, } 1}{ }^{\text {, Jinguo Ma }}{ }^{\text {Equal first author, } 2}{ }^{2}$, Xiaoying Xue ${ }^{\text {Corresp., } 1}{ }^{\text {, Guohui Wang }}{ }^{1}$, Tianfang Yan ${ }^{3}$, Linlin Su ${ }^{1}$, \\ Xuetao Han ${ }^{1}$, Huandi Zhou ${ }^{4}$, Liubing Hou ${ }^{4}$ \\ ${ }^{1}$ Department of Radiotherapy, The Second Hospital of Hebei Medical University, Shijiazhuang, China \\ 2 Department of Internal-Oncology, Hulun Buir People's hospital, Hulun Buir Medical school, Nationalities University of inner Mongolia,, Hulun Buir, China \\ 3 Department of Neurological Diagnosis and Restoration, Osaka University Graduate School of Medicine, Osaka, Japan \\ 4 Department of Central Laboratory, The Second Hospital of Hebei Medical University, Shijiazhuang, China \\ Corresponding Author: Xiaoying Xue \\ Email address: xxy0636@163.com
}

Background Aberrant DNA methylation of tumor suppressor genes is a common event in the development and progression of gastric cancer(GC). Our previous study showed NDRG1, which could suppress

cell invasion and migration, was frequently down-regulated by DNA methylation of its promoter in GC.

Purpose and Methods To analyze the relationship between the expression and DNA methylation of NDRG1 and DNA methyltransferase (DNMT) family. We performed a comprehensive comparison analysis using 407 patients including sequencing analysis data of GC from TCGA.

Results NDRG1 was down-regulated in GC, and was negatively correlative to DNMT1 ( $r=-0.11, p=0.03)$, DNMT3A $(r=-0.10, p=0.01)$, DNMT3B $(r=-0.01, p=0.88)$, respectively. Whereas, the DNA methylation of NDRG1 was positively correlative to DNMT family(DNMT1 $r=0.20, p<0.01$; DNMT3A $r=0.26$, $p<0.001$;DNMT3B $r=0.03, p=0.57$, respectively). NDRG1 expression was significantly inverse correlated with invasion depth ( $p=0.023$ ), but DNMT1 was significantly positive correlated with invasion depth ( $p$ $=0.049)$. DNMT3B was significantly correlated with the degree of tumor cell differentiation ( $p=0.030)$. However, there was no association between the expression of DNMT3A and clinicopathological features. The KM plotter showed that NDRG1(HR=0.95, 95\%Cl 0.8-1.12, $\mathrm{p}=0.53)$ and DNMT1 $(\mathrm{HR}=1.04,95 \% \mathrm{Cl}$ $0.88-1.23, p=0.67)$ had no association with prognosis of GC patients, while, DNMT3A $(p=0.0064)$ and $\operatorname{DNMT3B}(p=0.00025)$ displayed significantly association. But the overall survival of high expression of NDRG1 tended to be prolonged.

Conclusion These data suggest that down-regulation of NDRGlexpression in GC may be due to its promoter DNA methylation via DNMT family. The demethylating agent maybe a potential target drug for GC patients. 
1 DNMT family induces down-regulation of NDRG1 via DNA methylation and

2 clinicopathological significance in gastric cancer

3 Xiaojing Chang ${ }^{1 *}$, Jinguo $\mathrm{Ma}^{2 *}$, Xiaoying Xue ${ }^{1}$, Guohui Wang ${ }^{1}$, Tianfang Yan ${ }^{3}$, Linlin $\mathrm{Su}^{1}$,

4 Xuetao Han ${ }^{1}$, Huandi Zhou ${ }^{4}$, Liubing $\mathrm{Hou}^{4}$.

$5{ }^{1}$ Department of Radiotherapy, The Second Hospital of Hebei Medical University, Shijiazhuang,

6 China.

72 Department of Internal-Oncology, Hulun Buir People's hospital, Hulun Buir Medical school,

8 Nationalities University of inner Mongolia, Hulun Buir, China.

$9{ }^{3}$ Department of Neurological Diagnosis and Restoration, Osaka University Graduate School of

10 Medicine, Osaka, Japan.

$11{ }^{4}$ Department of Central Laboratory, The Second Hospital of Hebei Medical University,

12 Shijiazhuang, China

13 *Xiaojing Chang and Jinguo Ma have contributed equally to this manuscript.

14 Corresponding author: Xiaoying Xue, MD, PhD, Professor, Department of Radiotherapy, The

15 Second Hospital of Hebei Medical University, NO.215 Heping West Road, Shijiazhuang, China.

16 E-mail:xxy0636@163.com.

17 Running title:

18 The relationship of NDRG1 and DNMT family in gastric cancer. 
Abstract Background Aberrant DNA methylation of tumor suppressor genes is a common event in the development and progression of gastric cancer(GC). Our previous study showed NDRG1, which could suppress cell invasion and migration, was frequently down-regulated by DNA methylation of its promoter in GC. Purpose and Methods To analyze the relationship between the expression and DNA methylation of NDRG1 and DNA methyltransferase (DNMT) family. We performed a comprehensive comparison analysis using 407 patients including sequencing analysis data of GC from TCGA. Results NDRG1 was down-regulated in GC, and was negatively correlative to DNMT1 $(\mathrm{r}=-0.11, p=0.03)$, DNMT3A $(\mathrm{r}=-0.10, p=0.01)$, DNMT3B $(r=-0.01, p=0.88)$, respectively. Whereas, the DNA methylation of NDRG1 was positively correlative to DNMT family(DNMT1 $\mathrm{r}=0.20, \mathrm{p}<0.01 ; \quad$ DNMT3A $\mathrm{r}=0.26$, $\mathrm{p}<0.001 ; \mathrm{DNMT3B} \mathrm{r}=0.03, \mathrm{p}=0.57$, respectively). NDRG1 expression was significantly inverse correlated with invasion depth $(p=0.023)$, but DNMT1 was significantly positive correlated with invasion depth $(p=0.049)$. DNMT3B was significantly correlated with the degree of tumor cell differentiation $(p=0.030)$. However, there was no association between the expression of DNMT3A and clinicopathological features. The KM plotter showed that NDRG1(HR $=0.95$, 95\%CI 0.8-1.12, $\mathrm{p}=0.53)$ and DNMT1 $(\mathrm{HR}=1.04,95 \% \mathrm{CI} 0.88-1.23, \mathrm{p}=0.67)$ had no association with prognosis of GC patients, while, DNMT3A(p=0.0064) and DNMT3B $(p=0.00025)$ displayed significantly association. But the overall survival of high expression of NDRG1 tended to be 
45

46

47

prolonged. Conclusion These data suggest that down-regulation of NDRG1expression in GC may be due to its promoter DNA methylation via DNMT family. The demethylating agent maybe a potential target drug for GC patients.

Keywords: Gastric cancer, tumor suppressor gene, NDRG1, DNMT, DNA methylation, metastasis

\section{Introduction}

Gastric cancer (GC) is one of the most common digestive malignancies worldwide with the fourth most common cause of cancer-related death ${ }^{1,2}$. The poor prognosis and high recurrence rate are mainly due to lymph node metastasis at its early stage.

To date, a number of laboratories have shown that the occurrence of GC is usually caused by oncogenes activation and tumor suppressor genes(TSGs) inactivation. TSGs could suppress tumor cells migration and invasion, studies have shown that the inactivation of most TSGs are related to DNA methylation of $\mathrm{CpG}$, while the silencing of these genes possibly contributes to the development and progression of tumors ${ }^{3-5}$. There is increasing evidence that aberrant DNA promoter methylation of TSGs is a major event in the development and progression of $\mathrm{GC}^{6}$. Our previous studies showed NDRG1(N-myc downstream-regulated gene 1), a differentiation-related and tumor suppressor gene, which could suppress cancer cells invasion and migration, was frequently down-regulated by DNA methylation of its promoter in GC, while there were not any mutations in 16-exon sequences of NDRG1, and also not significant correlation with histone acetylation 7,8 .

DNA methylation of $\mathrm{CpG}$ islands, which is carried out by DNA methyltransferase (DNMT) enzymes, is the most widely and the best well studied epigenetic modification event, and leads to transcriptional gene silencing9. DNMT enzymes include DNMT1, DNMT2, DNMT3A, DNMT3B, and DNMT3L, among which DNMT1(a maintenance DNMT), DNMT3A and DNMT3B(de novo methyltransferases) are the most important ${ }^{10}$. In the current study, we focused on the relationship between NDRG1 and DNMT family(DNMT1, DNMT3A and DNMT3B) and 
71 their clinical significance and prognosis in GC by analyzing high-throughput data obtained from 72 TCGA.

\section{Materials and methods}

\section{The datasets of GC}

The data that we investigated the mRNA expression of NDRG1 and DNMT family members(DNMTs), and the DNA methylation of NDRG1 in GC were obtained from the data portal of TCGA(https://portal.gdc.cancer.gov/), which is a well-known cancer research project that collects and analyzes high-quality tumor samples and makes the related data available to researchers. We obtained the GC data set (March 1st, 2018 updated), which encompassed 407 gastric tumor samples, and of which 338 cases encompassed the data of DNA methylation of NDRG1. Total of 315 cases contained the complete clinicopathological information. GSE13911 data sets including 69 cases(including 38 GC tissues) of GC patients with microarray expression spectrum and corresponding clinical information were downloaded from the GEO (https://www.ncbi.nlm.nih.gov/geo/). Data from the TCGA and GEO database were free for using in any research study and do not require a separate consent from the participants. The present study was in compliance with the 1964 Declaration of Helsinki and its later amendments or comparable ethical standards.

\section{Prognostic analysis of NDRG1 and DNMT family via Kaplan-Meier (KM) plotter}

The prognostic values of overall survival (OS) for NDRG1 and DNMT family (RNAseq data) in GC $\quad(n=875) \quad$ were $\quad$ analyzed $\quad$ by $\quad$ plotter (http://kmplot.com/analysis/index.php? $\mathrm{p}=$ service\&cancer=gastric). The high/low expression of NDRG1 and each DNMT member were determined by the Median value. The results were displayed by hazard ratio (HR), 95\% confidence intervals $(95 \% \mathrm{CI})$ and log-rank p value (significant threshold was $<0.05$ ).

\section{Gastric Cells Culture and Real-Time PCR}

Human gastric cancer cell lines, SGC7901 and MKN45, and one immortalized normal gastric cell line, GES1 were cultured as described in our previous study ${ }^{7}$. 
98

99

100

101

102

103

104

105

106

107

108

109

110

111

112

113

114

115

116

117

118

119

120

121

122

123

Total RNA was isolated and reversely transcribed into cDNA as described previously ${ }^{7}$. The polymerase chain reaction (PCR) was performed in a volume of $20 \mathrm{ul}$ using MonAmp ${ }^{\mathrm{TM}}$ ChemoHS qPCR Mix (Monad Biotech Co., Ltd, China) with an initial denaturing at $95^{\circ} \mathrm{C}$ for 10 min, followed by 40 cycles of denaturing at $95^{\circ} \mathrm{C}$ for $10 \mathrm{~s}$, annealing for $10 \mathrm{~s}$ at $60^{\circ} \mathrm{C}$, extension for $30 \mathrm{~s}$ at $72^{\circ} \mathrm{C}$. The primers were shown in Table 1 . Relative gene expression to an internal GAPDH control and, hence, fold changes were calculated using the equation $2^{-\triangle \Delta \mathrm{Ct}}$ method.

\section{Protein Isolation and Western Blot}

Total cellular protein was extracted and western bolt was performed and anti-NDRG1 antibody was used as previous description ${ }^{7}$. Protein bands were scanned and quantified using densitometric software (Bio-Rad, California, USA). DNMT1, DNMT3A and DNMT3B antibodies at a dilution of 1:500 were purchased from Bioss, Beijing, China.

Statistics

All statistical analyses were conducted using SPSS 24.0 (Chicago, IL, USA) and R 3.6.1 (https://www.r-project. org/). R language and GraphPad Prism 7 (San Diego, CA, USA) were performed to draw plots. $\mathrm{X}^{2}$ test and Fisher's exact test were used to generate $\mathrm{P}$ values for clinicopathological parameters, Values with $p<0.05$ were considered as statistically significant.

\section{Results}

\section{The expression of NDRG1 and DNMT family in GC}

Total of $407 \mathrm{GC}$ cases(375 was GC tissues and 32 was nonmalignant gastric tissues)which included sequencing analysis data were downloaded from TCGA, then we firstly performed a expression analysis about the NDRG1 and DNMTs. As shown in Figure.1A-D, NDRG1 mRNA expression was down-regulated, but all of DNMT1、DNMT3A and DNMT3B were upregulated in $\mathrm{GC}$ tissues compared with normal tissues $(\mathrm{p}<0.05)$. To confirm these results, we further downloaded sequencing analysis data of GC from GEO which included $69 \mathrm{GC}$ cases(38 was GC tissues and 31 was nonmalignant gastric tissues), results were similar to that of TCGA, 
124 but the expression of NDRG1 and DNMT3A showed no statistical significance due to the small 125 sample size(Figure.2 A-D).

126 We also assessed the mRNA and protein levels of NDRG1 and DNMTs in one immortalized

127

128

129

130

131

132

133

134

135

136

137

138

139

140

141

142

143

144

145

146

147

148

149

150

normal gastric cell line, GES1 and two gastric cancer cell lines, SGC7901 and MKN45 by realtime PCR and western blot. Results showed DNMT1, DNMT3A and DNMT3B mRNA expression were up-regulated in SGC7901(1.63-fold,2.34-fold,2.14-fold,respectively) and MKN45(1.77-fold,3.62-fold,1.87-fold, respectively) cells compared with GES1(1 fold as the control). All of DNMTs mRNA level were negatively correlative to the expression NDRG1 which was shown down-regulated in GC cells(including SGC7901 and MKN45 cell lines) in our previous $\operatorname{study}^{7}(p<0.05$, Fig. 3A). Western blot analysis confirmed our data on NDRG1 and DNMTs mRNA expression(Fig. 3B). This was in accordance to the results of the relationship between NDRG1 and DNMT family mRNA expression from TCGA.

NDRG1 expression was negatively correlative to DNMT family, but its promoter methylation was positively correlative to the expression DNMT family in GC

We further performed a comprehensive comparison analysis about the mRNA expression of NDRG1 and DNMTs. As shown in Figure 4A-C, NDRG1 mRNA expression was negatively correlative to DNMT1（ $\mathrm{r}=-0.11, p=0.03)$, DNMT3A $(\mathrm{r}=-0.10, p=0.01), \quad$ DNMT3B $(\mathrm{r}=-$ $0.01, p=0.89$ ). Although the $\mathrm{R}$ value of comparison analysis was low, it still had statistical significance. NDRG1 was significantly associated with DNMT1 $(p=0.03)$ and DNMT3A $(p$ $=0.01)$, and DNMT3A showed the strongest association. However, there was no significant association between NDRG1 and DNMT3B $(p=0.89)$.

Among all of 407 GC cases, 338 cases encompassed the data of DNA methylation of NDRG1. Results showed DNA methylation of NDRG1 gene promoter was positively correlative to the expression DNMT family, and was significantly associated with DNMT1 $(\mathrm{r}=0.20, p=0.002$, Fig.4D) and DNMT3A ( $\mathrm{r}=0.26, p<0.001$, Fig.4E). However, no statistical significance was found between NDRG1 and DNMT3B ( $\mathrm{r}=0.03, p=0.57$, Fig. 4F). Although the $\mathrm{R}$ value of comparison analysis was not high, it still had statistical significance. It suggested that the down- 
151 regulation of NDRG1 in GC may be due to DNA methylation of its promoter via DNMT 152 family(DNMT1 and/or DNMT3A).

153

154

155

156

157

158

159

160

161

162

163

164

165

166

167

168

169

170

171

172

173

174

175

176

177

The association of NDRG1 and DNMT family with the clinicopathological parameters and prognosis of GC

The association of NDRG1 and DNMT family with the clinicopathological parameters of GC patients were shown in Table 2. Total of 315 cases which contained the complete clinicopathological and survival information were analyzed. The high and low expression of NDRG1 and DNMTs were based on the median value of their mRNA levels. We found NDRG1 expression was significantly inverse correlated with invasion depth $(p=0.023)$, whereas DNMT1 was statistically significantly positive correlated with invasion depth $(p=0.049)$, DNMT3B was significantly positive correlated with the degree of tumor cell differentiation $(p=0.030)$. There was no significant association between DNMT3A and the clinicopathological parameters of the GC patients (Table 2).

The prognostic values of NDRG1 and DNMTs in GC were assessed via the

KM plotter. Results showed that NDRG1(HR=0.95, 95\%CI 0.8-1.12, p=0.53) and DNMT1 $(\mathrm{HR}=1.04,95 \% \mathrm{CI} 0.88-1.23, \mathrm{p}=0.67)$ showed no significantly association with OS of GC patients. Whereas, DNMT3A(HR=1.35, 95\%CI 1.09-1.67, p=0.0064) and DNMT3B $(H R=1.37,95 \%$ CI 1.16-1.63, $\mathrm{p}=0.00025)$ were significantly correlated with prognosis of GC patients(Fig. 5A-D). But for median OS, it seemed that high expression of NDRG1 displayed good outcome(29.5 vs 27.8 months) compared with its low expression, while high expression of DNMT1, DNMT3A and DNMT3B showed poor prognosis.

\section{Discussion}

NDRG1, a differentiation-related gene, which belongs to NDRG family, is down-regulated in many tumors, and proposed as a metastasis suppressor gene ${ }^{11-14}$. Our previous studies confirmed the anti-cancer effect of NDRG1 in $\mathrm{GC}^{7,8,15}$. Gene network analysis shows that the 5 'end of the NDRG1 gene contains a large $\mathrm{CpG}$ island. It was reported that aberrant DNA methylation of $\mathrm{CpG}$ could lead to transcriptional gene silencing ${ }^{9}$. Some studies have confirmed 
178 the downregulation of NDRG1 caused by DNA methylation of $\mathrm{CpG}$ islands of its promoter in 179 breast cancer, prostate cancer cells and pancreatic cancer ${ }^{16-18}$. In GC, the down-regulation of 180 NDRG1 was also found to be regulated by DNA methylation of its promoter in our previous 181 study$^{7}$. DNA methylation, which is carried out by DNA methyltransferase enzymes, plays 182 important roles in the regulation of gene expression and the development and progression of tumors. DNMT family includes DNMT1, DNMT2, DNMT3A, DNMT3B, and DNMT3L, among which DNMT1, DNMT3A and DNMT3B are the most important members ${ }^{10}$. To date, not any studies reported the relationship of NDRG1 and DNMT family in GC.

In the current study, a total of $407 \mathrm{GC}$ cases which included sequencing analysis data of NDRG1 mRNA were analyzed. We found that all of DNMT1, DNMT3A and DNMT3B were up-regulated in GC, while, NDRG1 mRNA level was down-regulated in GC tissues. It is similar to our previous study ${ }^{7}$. Data from GEO and results of PCR and western blot analysis from GC cell lines confirmed these data from TCGA. Results of comparison analysis showed that NDRG1 expression was negatively correlative to DNMT1, DNMT3A and DNMT3B, and DNMT3A showed the strongest association. Although the $\mathrm{R}$ value of comparison analysis was not high, it still had statistical significance. But no significant association was found between NDRG1 and DNMT3B. This is the first time for analyzing the relationship between NDRG1 and DNMT family in GC. NDRG2, another member of NDRG family which includes four members NDRG1-4, was reported to be significantly decreased in human cancers, and in GC, H. pylori silenced Ndrg2 by activating the NF- $\mathrm{BB}$ pathway and up-regulating DNMT3b, promoting GC progression $^{19}$. Our current data were similar to those of NDRG2. Noteworthy, our data showed that aberrant DNA methylation of NDRG1 may be mainly due to the regulation of DNMT1 and DNMT3A.

We further investigated the association of DNA methylation of NDRG1 and DNMTs. Total 338 of all 407 GC cases encompassed the data of DNA methylation of NDRG1. Conversely, DNA methylation of NDRG1 was positively correlative to the expression of DNMTs, and was significantly associated with DNMT1 and DNMT3A.These results once again confirmed the 
205

206

207

208

209

210

211

212

213

214

215

216

217

218

219

220

221

222

223

224

225

226

227

228

229

230

231

relationship of NDRG1 mRNA expression and DNMTs in GC. Our previous study found that down-regulation of NDRG1 was regulated by DNA methylation of its promoter. DNA methylation of $\mathrm{CpG}$ islands leads to transcriptional gene silencing which is carried out by DNMTs. Thus, our current data suggests that down-regulation of NDRG1 in GC may be due to DNA methylation of NDRG1 gene promoter via DNMT family. Whereas, NDRG1 expression was significantly inverse correlated with invasion depth, and DNMT1 was positively correlated with invasion depth. DNMT3B was significantly positive correlated with the degree of tumor cell differentiation. But, KM plotter showed no association between NDRG1and DNMT1 with the prognosis of GC patients. While, high expression of DNMT3A and DNMT3B were significantly correlated with poor prognosis of GC patients. But for median survival, it seemed that high expression of NDRG1 displayed good outcome and the OS tended to be prolonged. These results were mainly based on the data of bioinformatics, the current data remain suggest that high NDRG1 expression and low DNMTs expression could suppress cell invasion and migration, may improve the rate of recurrence and metastasis. Our previous study had showed GC patients with high expression of NDRG1 had better overall survival rate than those with low NDRG1 expression ${ }^{8}$. So large sample data of immunohistochemistry, western blot and RT-PCR of GC tissues were urgently needed.

DNMT inhibitor, including azacitidine, 5-aza-2'-deoxycytidine (5-Aza-Dc, decitabine), guadecitabine, hydralazine, procaine, MG98 and/or zebularine, among which 5-Aza-Dc is the most commonly used, could cause demethylating and reactivate the expression of TSGs, then suppress the metastasis of tumor cells ${ }^{20-22}$. NDRG1 expression was found to be increased after

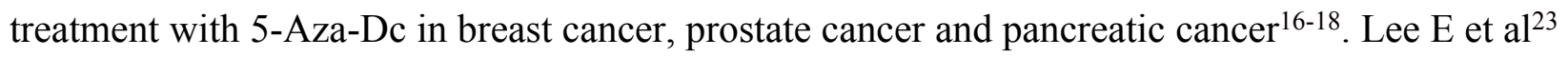
found that the epidrug 5-Aza could up-regulated NDRG1 expression by reduction of suppressive histone marks, $\mathrm{H} 3 \mathrm{~K} 9 \mathrm{me} 3$ and $\mathrm{H} 3 \mathrm{~K} 27 \mathrm{me} 3$ on NDRG1 promoter in prostate cancer.

In the current study, the aberrant DNA methylation of NDRG1 was found to maybe significantly associated with DNMT family, especially DNMT1 and DNMT3A. Our previous study showed that the down-regulation of NDRG1 was also found to be regulated by DNA 
232 methylation of its promoter and 5-Aza-Dc could up-regulate its expression in GC cells. It 233 suggests that the demethylating agent maybe a potential target drug in GC. Up to now, 234 demethylating treatment has been used for the treatment of myeloid leukemias ${ }^{24,}{ }^{25}$. In vitro 235 studies of solid tumors, demethylating agents combined chemotherapy and/or immunotherapy 236 could enhance the therapeutic effect in cancer cells ${ }^{26-29}$. In a case report, azacitidine was used to 237 treat a 57-year-old woman newly diagnosed with MDS during palliative chemotherapy for 238 metastatic breast cancer, azacitidine showed promising effects for MDS, and also stabilized the 239 patient's lung and lymph node metastases without any major toxicity ${ }^{30}$. Now, An increasing 240 number of clinical trials of demethylating treatment is ongoing, some have presented exciting 241 effects $^{31-34}$ (Table 3). A Phase I study showed the security of decitabine treated by hepatic 242 arterial infusion, and a dose level of $20 \mathrm{mg} / \mathrm{m} 2 /$ day on five consecutive days every 4 weeks could 243 be considered for further investigation in combinatorial immunotherapy regimens in patients 244 with Unresectable Liver-predominant Metastases From Colorectal Cancer ${ }^{33}$. In a phase I/II 245 clinical trial, 15 patients with metastatic castration-resistant prostate cancer (mCRPC) were 246 enrolled in phase I which was dose climbing experiment of 5-Aza-Dc, and 7 patients were enrolled in phase II. In phase I, no dose-limiting toxicity was observed, and most patients were well tolerated. The highest level reached was 5 -Aza-Dc with $150 \mathrm{mg} / \mathrm{m} 2$ daily for 5 days followed by docetaxel with $75 \mathrm{mg} / \mathrm{m} 2$ on day 6 , which was considered as the recommended phase II dose. In phase II, 6 patients received the recommended phase II dose during 46 cycles. Two episodes of Grade 3 hematologic and three Grade 3 nonhematologic toxicities were observed, and one patient died from neutropenic sepsis. Subsequently, 5-Aza was reduced to $75 \mathrm{mg} / \mathrm{m} 2$, and no treatment-related $>$ Grade 3 toxicities was observed in one patient. In this clinical trial, PSA response was observed in 10 of 19 (52.6 \%) patients, and the median duration of response was 20.5 weeks. Kaplane-Meier estimate of median PFS was 4.9 months, and median OS was 19.5 months, which were both favorable ${ }^{33}$. It suggests that the combination of azacitidine and chemotherapy is active in mCRPC patients, and it maybe a new treatment in 258 future for tumors. 
259

260

261

262

263

264

265

266

267

268

269

270

271

272

273

274

275

276

277

278

279

280

281

282

283

284

285

286

287

288

289

290

291

292

This study is mainly based on the data of bioinformatics, large sample data of immunohistochemistry, western blot and RT-PCR of GC tissues are urgently needed. We will further detect and confirm the relationship of NDRG1 and DNMTs in GC tissues and in vivo and vitro experiments.

In conclusion, the current data showed that the down-regulated and its aberrant DNA methylation of NDRG1 which could suppress cell invasion and migration, maybe mainly regulated by DNMT family in GC, especially DNMT1 and DNMT3A. Furthermore, the demethylating agent 5-Aza-Dc, a DNMT inhibitor, maybe a potential target drug. Therefore, further clinical studies are warranted to evaluate and confirm the effect of demethylating treatment in GC.

Acknowledgments This study is supported in part by a grant from the National Natural Science Foundation of Hebei province of China(\#H2018206180).

Disclosures No conflicts of interest are declared by the authors.

\section{References:}

1. Sung H, Ferlay J, Siegel RL, Laversanne M, Soerjomataram I, Jemal A, Bray F. Global cancer statistics 2020: GLOBOCAN estimates of incidence and mortality worldwide for 36 cancers in 185 countries. CA Cancer J Clin 2021; 71(3):209-249.

2. Ferlay J, Colombet M, Soerjomataram I, Mathers C, Parkin DM, Piñeros M, Znaor A, Bray F. Estimating the global cancer incidence and mortality in 2018:GLOBOCAN sources and methods. INT J CANCER 2019; 144(8): 1941-1953.

3. Song J, Teplova M, Ishibe-Murakami S, Patel DJ. Structure-based mechanisitic insights into DNMT1-mediated maintenance DNA methylation. SCIENCE 2012; 335(6069): 709-712.

4. Celarain N, Tomas-Roig J. Aberrant DNA methylation profile exacerbates inflammation and neurodegeneration in multiple sclerosis patients. J Neuroinflammation 2020; 17: 21.

5. Hackett JA, Surani MA. DNA methylation dynamics during the mammalian life cycle. Philos Trans R Soc Lond B Biol Sci 2013; 368(1609): 20110328.

6. Qu Y, Dang S, Hou P. Gene methylation in gastric cancer. CLIN CHIM ACTA 2013; 424: 53-65. 
293

294

295

296

297

298

299

300

301

302

303

304

305

306

307

308

309

310

311

312

313

314

315

316

317

318

319

320

321

322

323

324

325

326

327

328

329

330

7. Chang X. Association of NDRG1 gene promoter methylation with reduced NDRG1 expression in gastric cancer cells and tissue specimens. CELL BIOCHEM BIOPHYS 2013; 66(1): 93-101.

8. Chang X. NDRG1 expression is related to the progression and prognosis of gastric cancer patients through modulating proliferation, invasion and cell cycle of gastric cancer cells. $M O L$ BIOL REP 2014; 41(9): 6215-6223.

9. Lyko F. The DNA Methyltransferase Family: A Versatile Toolkit for Epigenetic Regulation. NAT REV GENET 2018; 19(2): 81-92.

10. Baylin SB, Jones PA. A decade of exploring the cancer

epigenome-Biological and translational implications. NAT REV CANCER 2011; 11(10): 726734.

11. Wang J, Lv W, Lin Z, Wang X, Bu J, Su Y. Hsa_circ_0003159 inhibits gastric cancer progression by regulating miR-223-3p/NDRG1 axis. CANCER CELL INT 2020; 20: 57.

12. Chen Z, Sun J, Li T, Liu Y, Gao S, Zhi X, Zheng M. Iron chelator-induced up-regulation of Ndrg1 inhibits proliferation and EMT process by targeting Wnt/ $\beta$-catenin pathway in colon cancer cells. Biochem Biophys Res Commun 2018; 506(1): 114-121.

13. Park KC, Paluncic J, Kovacevic Z, Richardson DR. Pharmacological targeting and the diverse functions of the metastasis suppressor, NDRG1, in cancer. Free 2019; S0891-5849(19): 30254-30256.

14. Menezes SV, Kovacevic Z, Richardson DR. The metastasis suppressor NDRG1 downregulates the epidermal growth factor receptor via a lysosomal mechanism by upregulating mitogen-inducible gene 6. J BIOL CHEM 2019; 294(11): 4045-4064.

15. Chang X, Xu X, Xue X, Ma J, Li Z, Deng P, Chen J, Zhang S, Zhi Y, Dai D. NDRG1 controls gastric cancer migration and invasion through regulating MMP-9. PATHOL ONCOL RES 2016; 22(4): 789-796.

16. Han LL. Aberrant NDRG1 methylation associated with its decreased expression and clinicopathological significance in breast cancer. J BIOMED SCI 2013; 20: 52.

17. Li Y. Downregulation of N-myc downstream regulated gene 1 caused by the methylation of 
$331 \mathrm{CpG}$ islands of NDRG1 promoter promotes proliferation and invasion of prostate cancer cells. 332 INT J ONCOL 2015; 47(3): 1001-1008.

333

334

335

336

337

338

339

340

341

342

343

344

345

346

347

348

349

350

351

352

353

354

355

356

357

358

359

360

361

362

363

364

365

366

367

368

369

370

18. Angst E. Epigenetic regulation affects N-myc downstream-regulated gene 1 expression indirectly in pancreatic cancer cells. PANCREAS 2010; 39(5): 675-679.

19. Ling ZQ. Ndrg2 promoter hypermethylation triggered by helicobacter pylori infection correlates with poor patients survival in human gastric carcinoma. Oncotarget 2015; 6(10): 8210-8225.

20. Cheishvili D. DNA Demethylation and Invasive Cancer: Implications for Therapeutics. $B r J$ Pharmacol 2015; 172(11): 2705-2715.

21. Zhou Z. DNA Methyltransferase Inhibitors and their Therapeutic Potential. CURR TOP MED CHEM 2018; 18(28): 2448-2457.

22. Rygiel Katarzyna. Epigenetic therapies in patients with solid tumors: Focus on monotherapy with deoxyribonucleic acid methyltransferase inhibitors and histone deacetylase inhibitors. 2019; 15(5): 961-970.

23. Lee E, Wang J, Jung Y, Cackowski FC, Taichman RS. Reduction of two histone marks, $\mathrm{H} 3 \mathrm{k} 9 \mathrm{me} 3$ and $\mathrm{H} 3 \mathrm{k} 27 \mathrm{me} 3$ by epidrug induces neuroendocrine differentiation in prostate cancer. $J$ CELL BIOCHEM 2018; 119(4): 3697-3705.

24. Sato T, Issa JJ, Kropf P. DNA Hypomethylating Drugs in Cancer Therapy. Cold Spring Harb Perspect Med 2017; 7(5): a26948.

25. Dombret H, Itzykson R. How and when to decide between epigenetic therapy and chemotherapy in patients with AML. Hematology Am Soc Hematol Educ Program 2017; 2017(1): 45-53.

26. Li XY, Wu JZ, Cao HX, Ma R, Wu JQ, Zhong YJ, Feng JF. Blockade of DNA methylation enhances the therapeutic effect of gefitinib in non-small cell lung cells

. ONCOL REP 2013; 29(5): 1975-1982.

27. Lou YF, Zou ZZ, Chen PJ, Huang GB, Li B, Zheng DQ, Yu XR, Luo XY. Combination of Gefitinib and DNA Methylation Inhibitor Decitabine Exerts Synergistic Anti-Cancer Activity in Colon Cancer Cells. PLOS ONE 2014; 9(5): e97719.

28. Vijayaraghavalu S, Labhasetwar V. Nanogel-mediated delivery of a cocktail of epigenetic 
371 drugs plus doxorubicin overcomes drug resistance in breast cancer cells. Drug Deliv Transl Res

$372 \quad 2018 ; 8(5): 1289-1299$.

373

374

375

376

377

378

379

380

381

382

383

384

385

386

387

388

389

390

391

392

393

394

395

396

397

398

399

400

401

402

403

404

405

406
29. Luo N, Sugiura A, Balko JM. Therapeutic potential of DNA methyltransferase inhibitors with immune checkpoint inhibitor therapy in breast cancer. Cell Stress 2018; 2(3): 69-71.

30. Baek DW, Lee SJ, Sohn SK, Moon JH, Chae YS. Clinical Effects of Hypomethylating Agents in Patients with Newly Diagnosed Myelodysplastic Syndrome Who Received DNADamaging Chemotherapy for Metastatic Breast Cancer. J Breast Cancer. 2019; 22(4): 647-652.

31. Linnekamp JF, Butter R, Spijker R, Medema JP, van Laarhoven HWM. Clinical and biological effects of demethylating agents on solid tumours - A systematic review. CANCER TREAT REV 2017; 54: 10-23.

32. Perrard J, Morel A, Meznad K, Paget-Bailly P, Dalstein V, Guenat D, Mourareau C, Clavel C, Fauconnet S, Baguet A, Mougin C, Pretet JL. DNA Demethylation Agent 5azadC Downregulates HPV16 E6 Expression in Cervical Cancer Cell Lines Independently of TBX2 Expression. ONCOL LETT 2020; 19(1): 1074-1081.

33. Singal R, Ramachandran K, Gordian E, Quintero C, Zhao W, Reis IM. Phase I/II study of azacitidine, docetaxel, and prednisone in patients with metastatic castration-resistant prostate cancer previously treated with docetaxel-based therapy. Clin Genitourin Cancer 2015; 13(1): 2231.

34. Jansen YJL, Verset G, Schats K, Van Dam PJ, Seremet T, Kockx M, Van Laethem JB, B. Phase I clinical trial of decitabine (5-aza-2'-deoxycytidine) administered by hepatic arterial infusion in patients with unresectable liver-predominant metastases. ESMO Open. 2019; 4(2): e464. 
417 Caption:

418 Figure.1A-D, The mRNA expression of NDRG1, DNMT1, DNMT3A and DNMT3B in GC 419 from the data of TCGA.

420 Figure.2A-D The mRNA expression of NDRG1, DNMT1, DNMT3A and DNMT3B in GC from 421 the data of GEO. ${ }^{*} \mathrm{p}<0.05 ; * * * \mathrm{p}<0.001$; ns, no statistical significance.

422 Figure.3 The expression levels of NDRG1 and DNMTs in GES1 and two gastric cancer cell lines, SGC7901 and MKN45.A, the mRNA level of NDRG1 and DNMTs; B. the protein level of 424 NDRG1 and DNMTs $(* \mathrm{p}<0.05, * * \mathrm{p}<0.01)$.

425 Figure.4 Association between NDRG1 and DNMTs. A-C, NDRG1 mRNA expression was 426 negatively correlative to DNMT1, DNMT3A and DNMT3B. D-F, DNA methylation of NDRG1 427 gene promoter was positively correlative to the expression DNMT family.

428 Figure.5 Survival curves of NDRG1 and DNMT family in GC via the Kaplan-Meier 429 (KM) plotter. Red: high expression; black: low expression; HR: hazard ratio.

430 Table.1 primers of NDRG1 and DNMT family

431 Table.2 Clinicopathological parameters of NDRG1 and DNMTs mRNA expression from 432 TCGA cohort

433 Table.3 Ongoing clinical trials evaluating decitabine drug in solid tumors 434 
Figure 1

Figure.1A-D The mRNA expression of NDRG1, DNMT1, DNMT3A and DNMT3B in GC.

A-D The mRNA expression of NDRG1, DNMT1, DNMT3A and DNMT3B in GC.

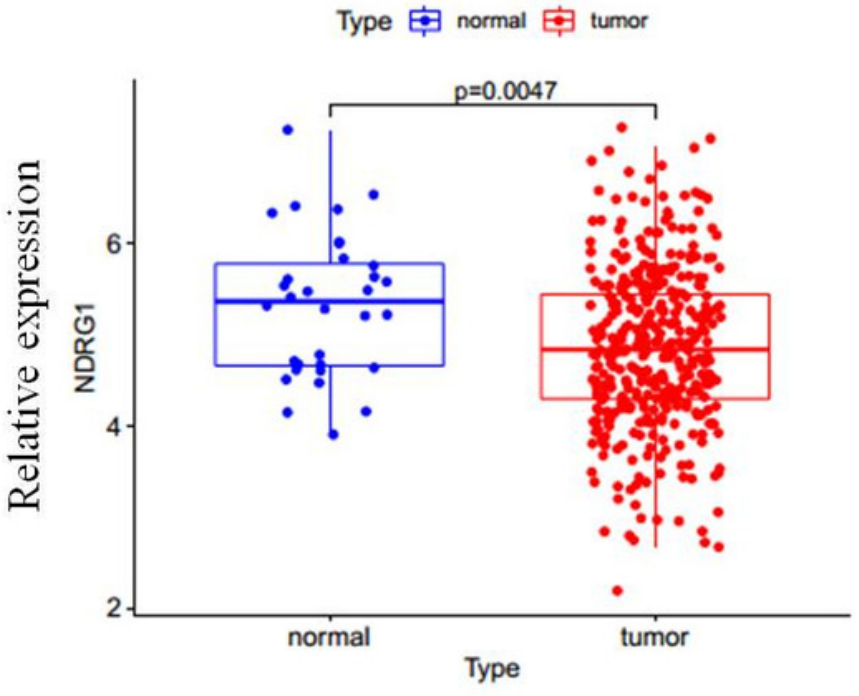

A

Type 审 normal 审 tumor

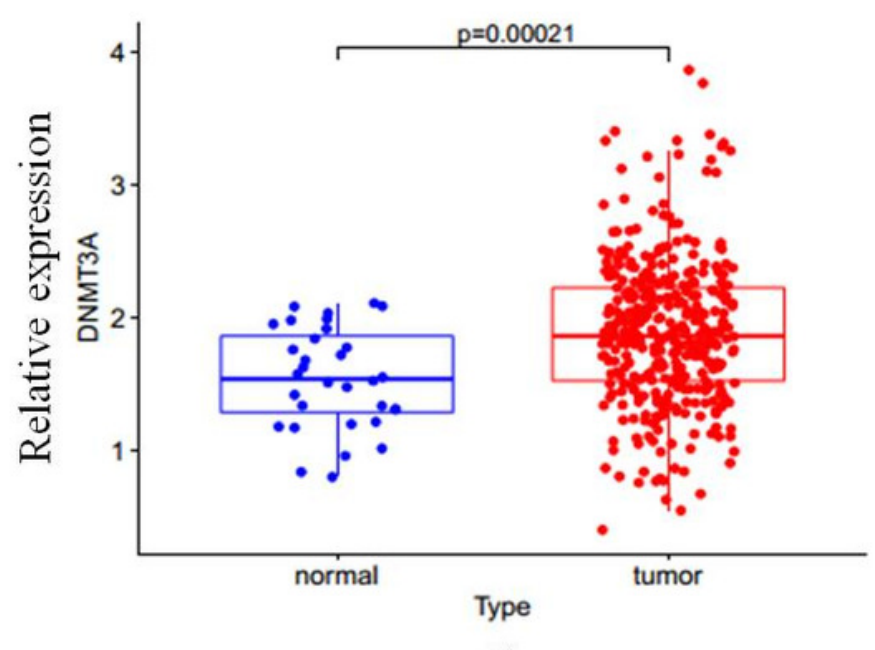

$\mathrm{C}$

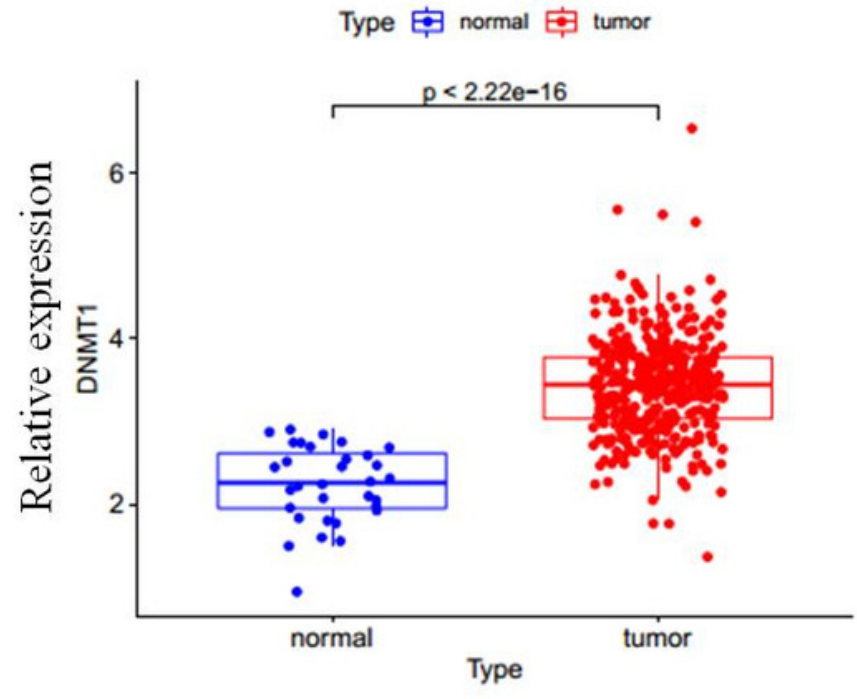

B

Type 후 normal 후 tumor

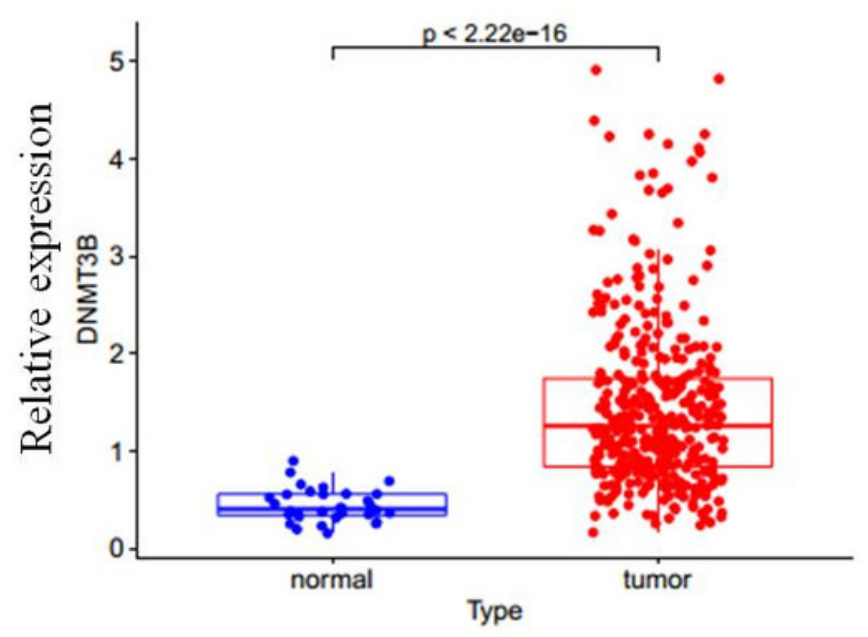

$\mathrm{D}$ 
Figure 2

Figure.2A-D The mRNA expression of NDRG1, DNMT1, DNMT3A and DNMT3B in GC from the data of GEO. $* p<0.05 ; * * * p<0.001 ; n s$, no statistical significance.

Figure.2A-D The mRNA expression of NDRG1, DNMT1, DNMT3A and DNMT3B in GC from the data of GEO. $* p<0.05 ; * * * p<0.001 ;$ ns, no statistical significance.

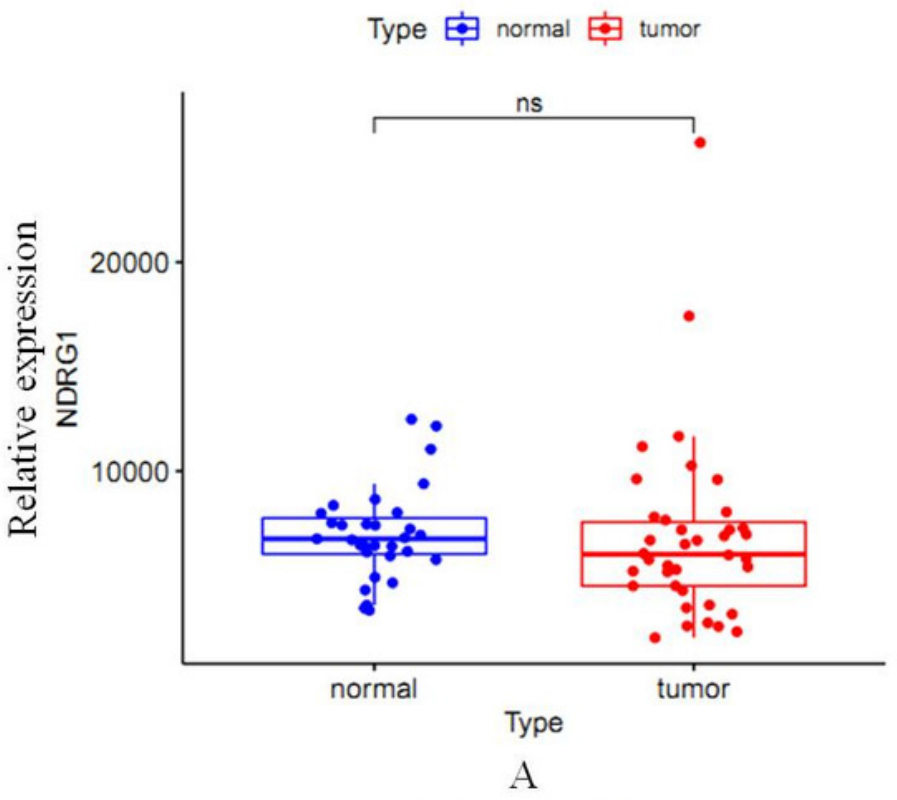

Type 审 normal 审 tumor

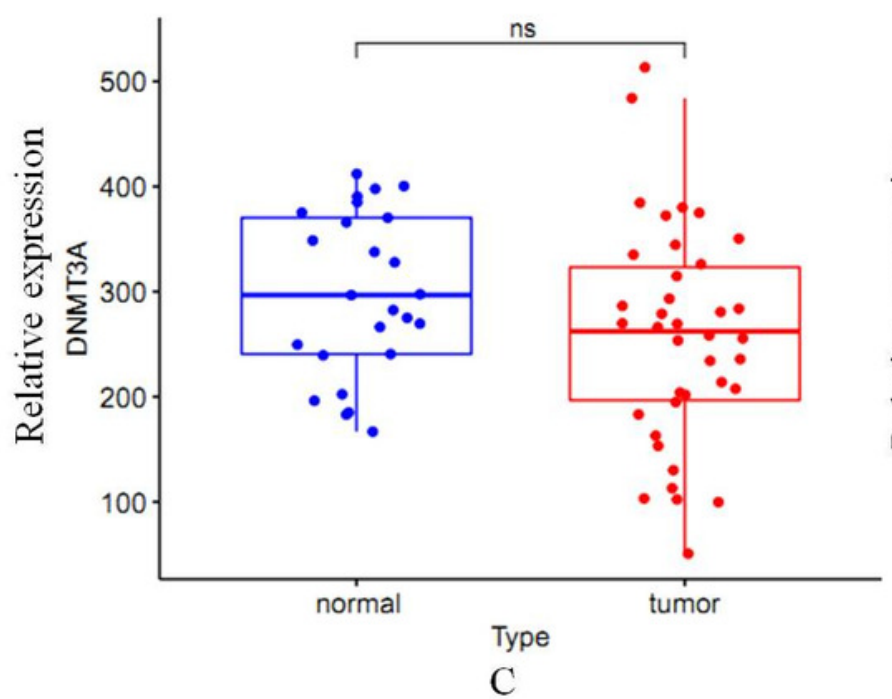

Type 审 normal 审 tumor

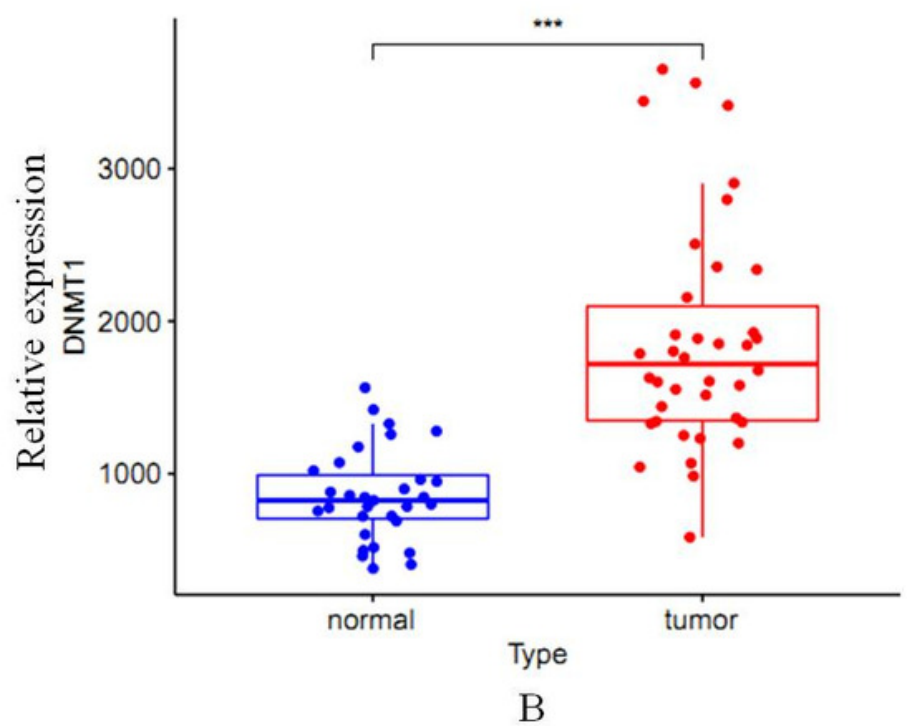

Type 审 normal 하 tumor

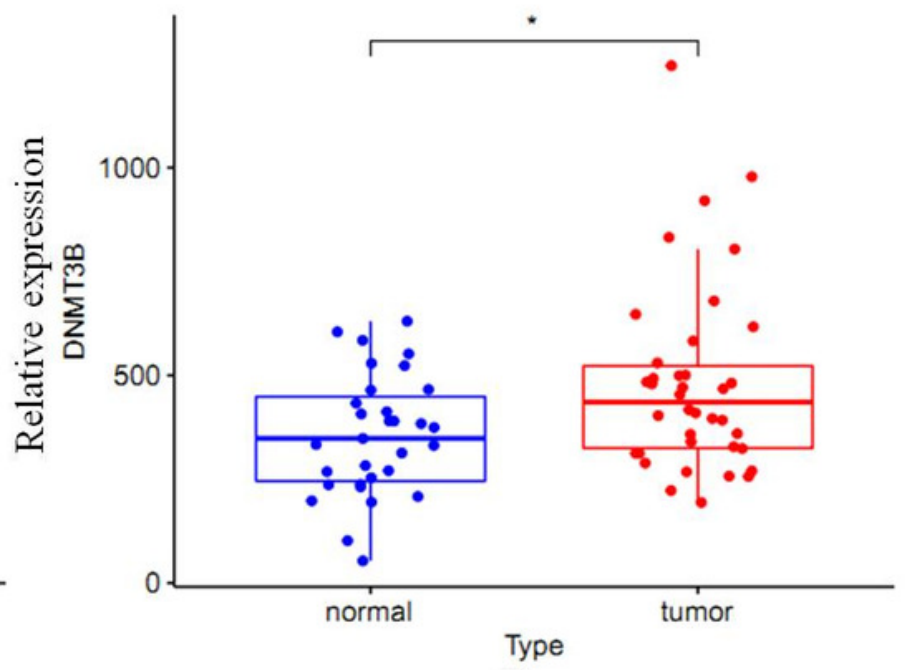

D 


\section{Figure 3}

Figure.3 The expression levels of NDRG1 and DNMTs in GES1 and two gastriccell lines, SGC7901 and MKN45.A, the mRNA level of NDRG1 and DNMTs; B. theprotein level of NDRG1 and DNMTs $(* p<0.05$, $* * p<0.01)$.

Figure.3 The expression levels of NDRG1 and DNMTs in GES1 and two gastric cell lines, SGC7901 and MKN45.A, the mRNA level of NDRG1 and DNMTs; B. the protein level of NDRG1 and DNMTs(* $p<0.05$, $* * p<0.01)$.

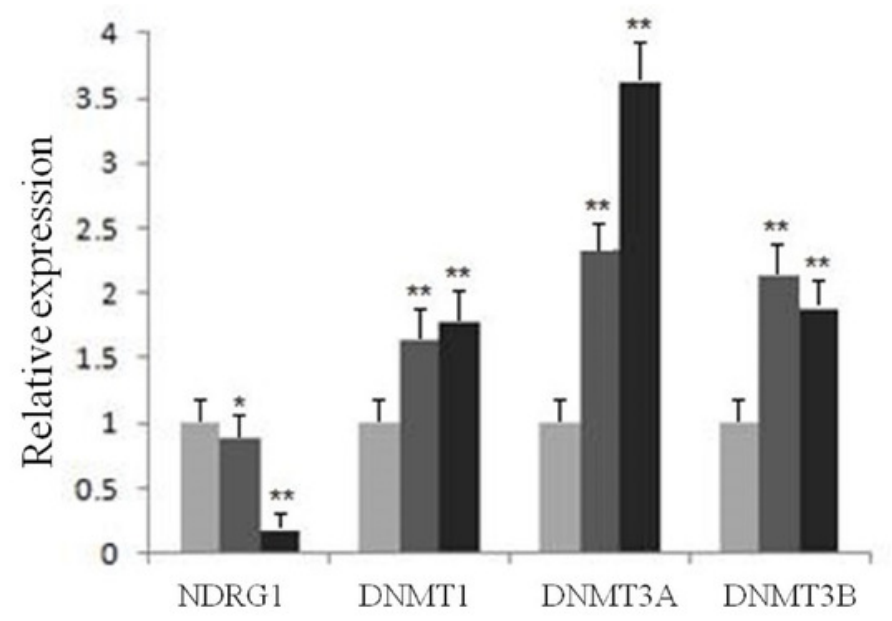

A

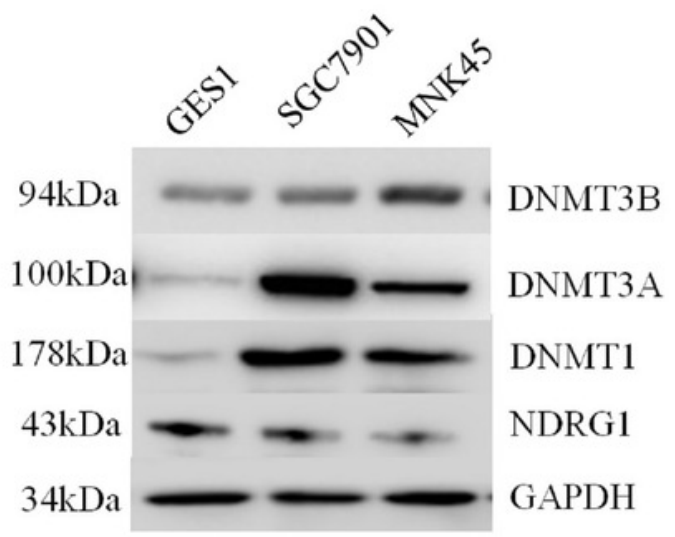

B 
Figure 4

Figure.4 Association between NDRG1 and DNMTs. A-C, NDRG1 mRNA expression was negativelycorrelative to DNMT1, DNMT3A and DNMT3B. D-F, DNA methylation of NDRG1 gene promoterwas positively correlative to the expression DNMT family.

Figure.4 Association between NDRG1 and DNMTs. A-C, NDRG1 mRNA expression was negatively correlative to DNMT1, DNMT3A and DNMT3B. D-F, DNA methylation of NDRG1 gene promoter was positively correlative to the expression DNMT family.

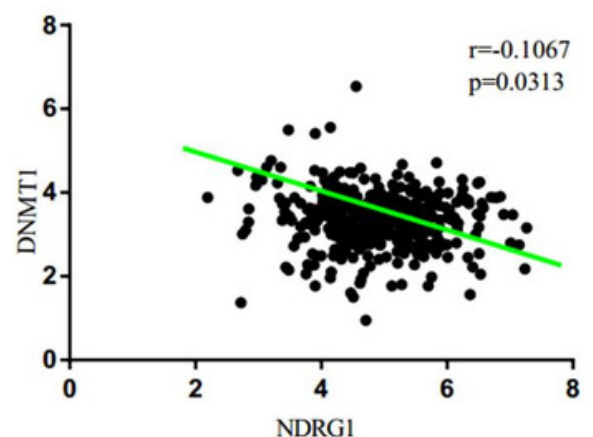

A

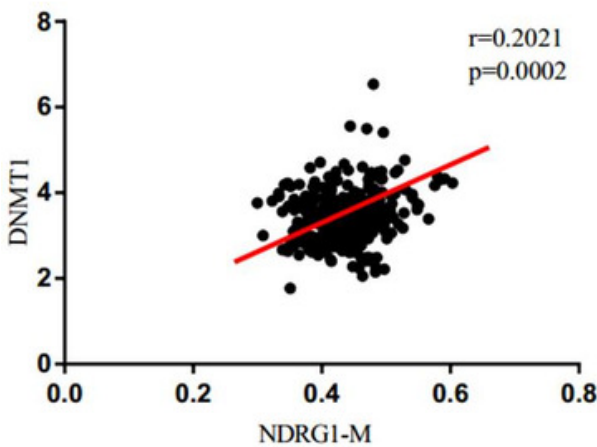

D

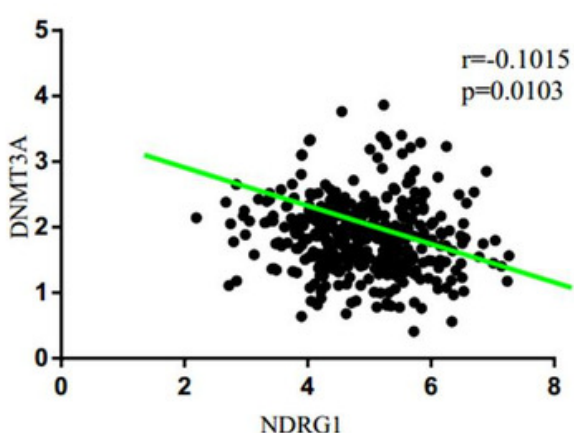

$\mathrm{B}$

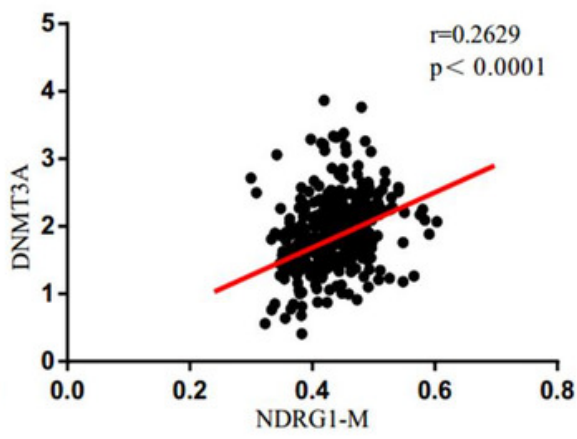

E

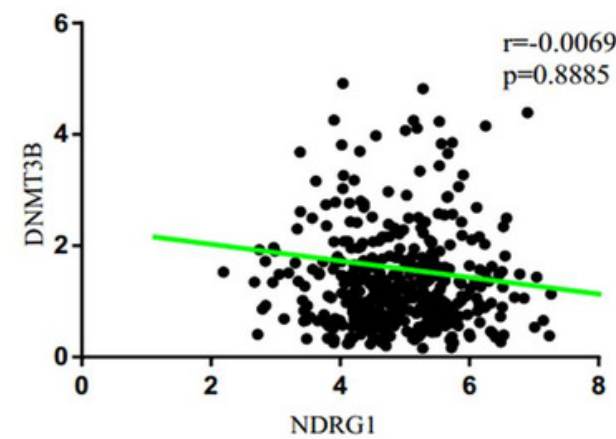

$\mathrm{C}$

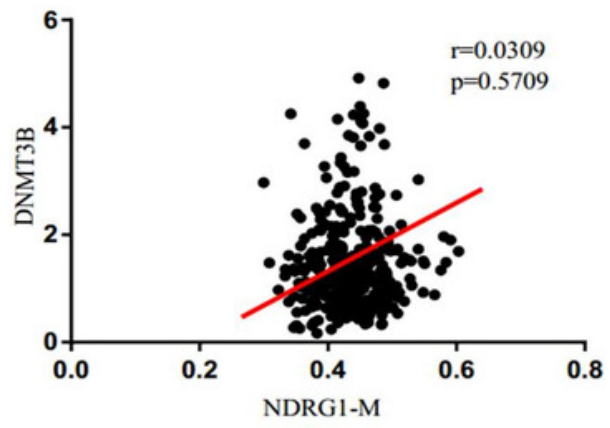

$\mathrm{F}$ 


\section{Figure 5}

Figure.5 Survival curves of NDRG1 and DNMTfamily in GC via the Kaplan-Meier (KM) plotter. Red: high expression; black: low expression;HR: hazard ratio.

Figure.5 Survival curves of NDRG1 and DNMT family in GC via the Kaplan-Meier (KM) plotter. Red: high expression; black: low expression; HR: hazard ratio. 
NDRG1

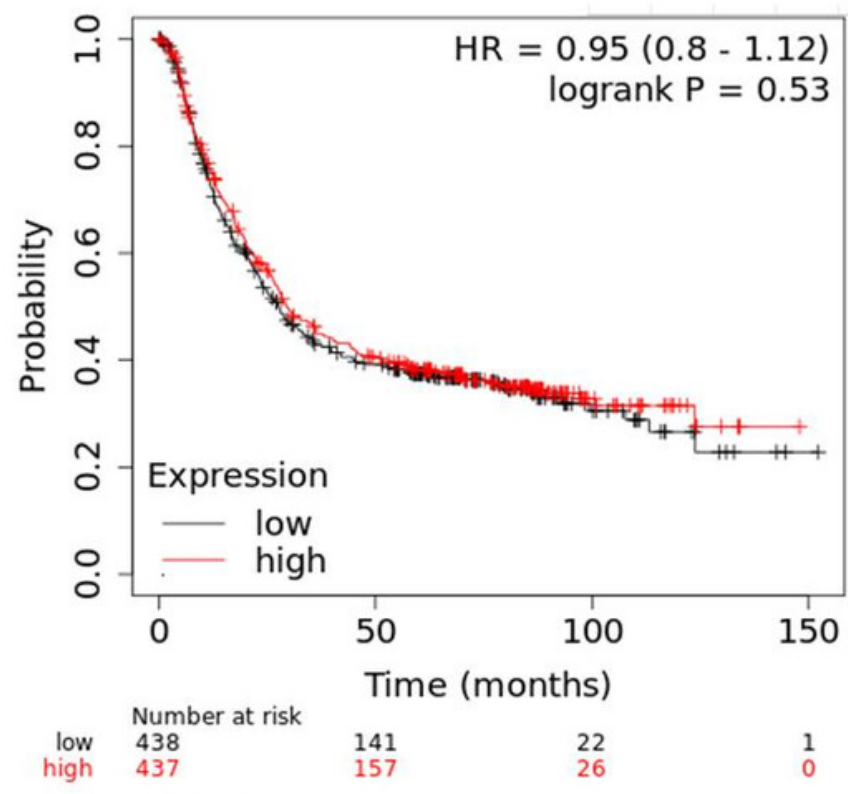

Median survival

\begin{tabular}{|l|l|}
\hline Low expression cohort (months) & High expression cohort (months)
\end{tabular} \begin{tabular}{|c|r|}
\hline Low expression cohort (months) & High expression co \\
\hline 27.8 & 29.5 \\
\hline
\end{tabular}

DNMT3A

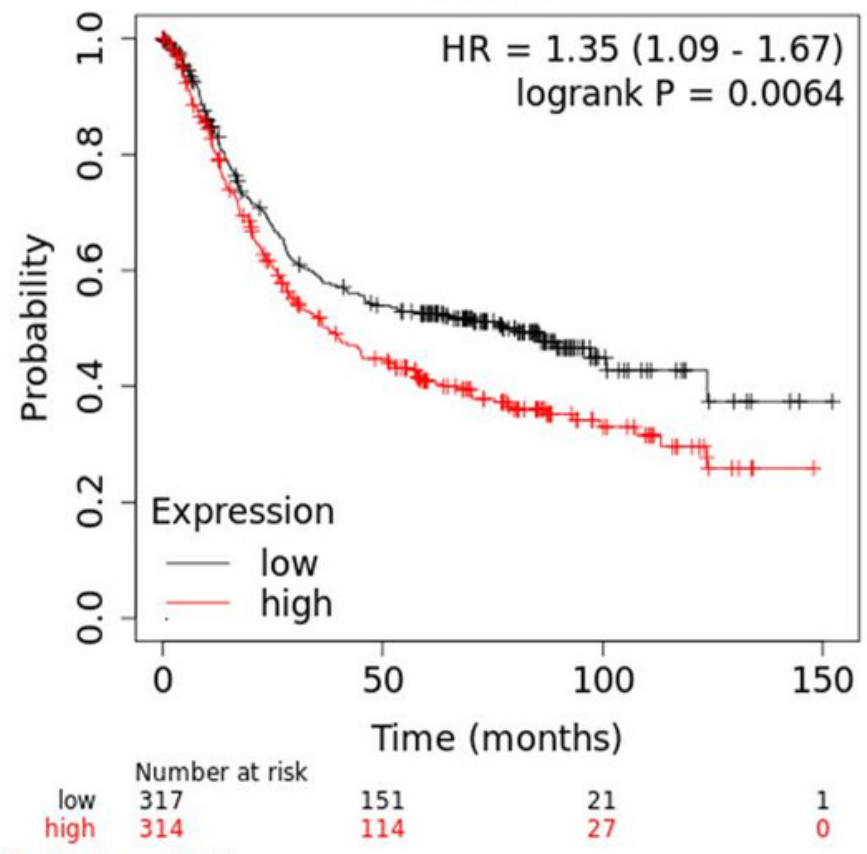

Median survival

\begin{tabular}{|l|l|}
\hline Low expression cohort (months) & High expression cohort (months)
\end{tabular}

\section{DNMT1}

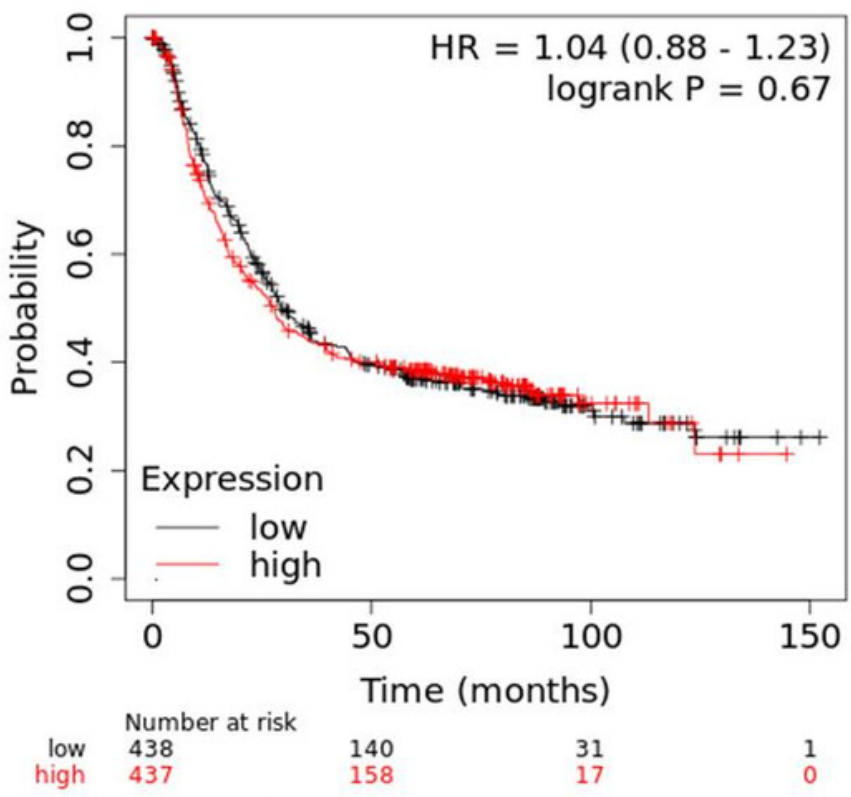

Median survival

Low expression cohort (months) High expression cohort (months)

\begin{tabular}{|c|c|}
\hline 29.5 & 27.8 \\
\hline
\end{tabular}

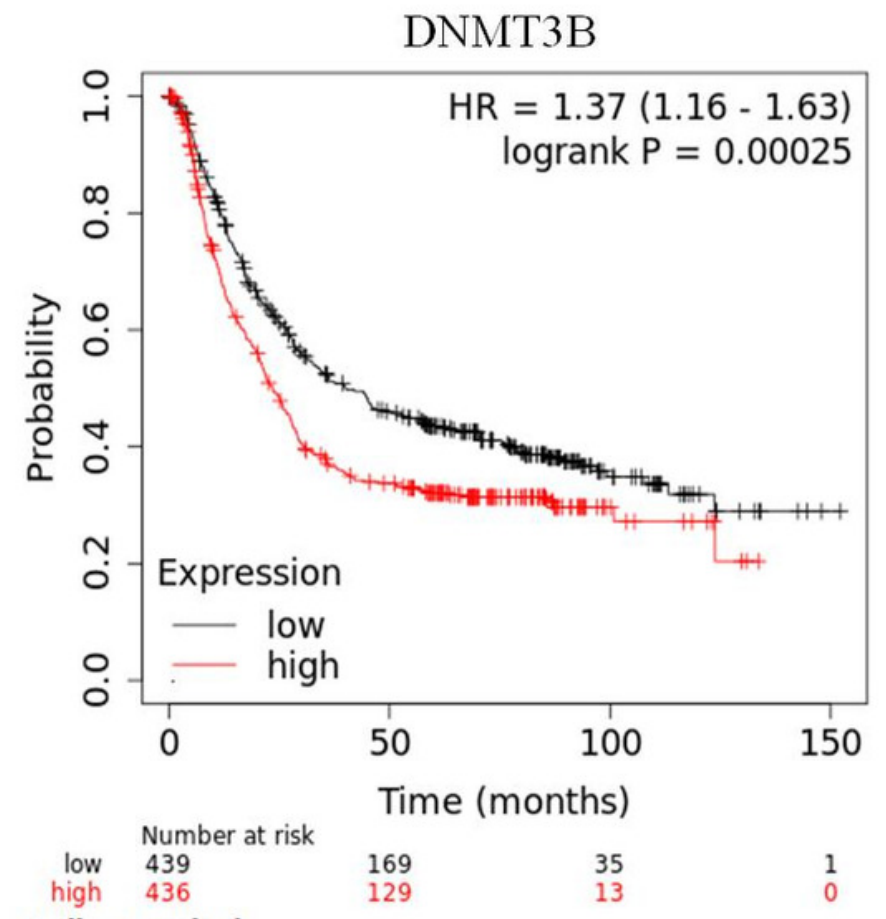

Median survival

\begin{tabular}{|c|c|}
\hline Low expression cohort (months) & High expression cohort (months) \\
\hline 40.1 & 23.5 \\
\hline
\end{tabular}


Table $\mathbf{1}$ (on next page)

Table.1 primers of NDRG1 and DNMT family

Table.1 primers of NDRG1 and DNMT family 
1 Table.1 primers of NDRG1 and DNMT family

\begin{tabular}{ll}
\hline Gene & primer \\
\hline NDRG1 & 5'-AGTTGGAGTAAGTGTTTGTGAGT-3' (sense) \\
& 5'-AAACAAAAACAAAAATAACACCACT-3' (antisense) \\
DNMT1 & 5'-AGGCGGCTCAAAGATTTGGAA-3'( 'sense) \\
& 5'-GCAGAAATTCGTGCAAGAGATTC-3' (antisense) \\
DNMT3A & 5'-CCGATGCTGGGGACAAGAAT-3' (sense) \\
& 5'-CCCGTCATCCACCAAGACAC-3' (antisense) \\
DNMT3B & 5'-AGGGAAGACTCGATCCTCGTC-3' (sense) \\
& 5'-GTGTGTAGCTTAGCAGACTGG-3' (antisense) \\
GAPDH & 5'-CATGAGAAGTATGACAACAGCCT-3' (sense) \\
& 5'-AGTCCTTCCACGATACCAAAGT-3' (antisense) \\
\hline
\end{tabular}

2 


\section{Table 2 (on next page)}

Table.2 Clinicopathological parameters of NDRG1 and DNMTsmRNA expression from TCGA cohort 
1 Table 2. Clinicopathological parameters of NDRG1 and DNMTs mRNA expression from TCGA cohort

\begin{tabular}{|c|c|c|c|c|c|c|c|c|c|}
\hline \multirow[t]{2}{*}{ Variable } & \multirow{2}{*}{$\begin{array}{l}\text { Patients } \\
\text { (n) }\end{array}$} & \multicolumn{2}{|l|}{ NDRG1 } & \multicolumn{2}{|c|}{ DNMT1 } & \multicolumn{2}{|c|}{ DNMT3A } & \multicolumn{2}{|c|}{ DNMT3B } \\
\hline & & Low(High) & $\mathrm{p}$ & Low(High) & $\mathrm{p}$ & Low(High) & $\mathrm{p}$ & Low(High) & $\mathrm{p}$ \\
\hline \multicolumn{10}{|l|}{ Gender } \\
\hline Female & 120 & $61(59)$ & 0.908 & $56(64)$ & 0.355 & $56(64)$ & 0.355 & $61(59)$ & 0.817 \\
\hline Male & 195 & $97(98)$ & & $102(93)$ & & $102(93)$ & & $96(99)$ & \\
\hline \multicolumn{10}{|l|}{ Age } \\
\hline$<60$ & 100 & $52(48)$ & 0.717 & $47(53)$ & 0.469 & $50(50)$ & 1.000 & $49(51)$ & 0.904 \\
\hline$\geqslant 60$ & 215 & 106(109) & & 111(104) & & $108(107)$ & & $108(107)$ & \\
\hline \multicolumn{10}{|l|}{ Stage } \\
\hline I & 42 & $18(24)$ & 0.297 & $23(19)$ & 0.881 & $26(16)$ & 0.190 & $20(22)$ & 0.814 \\
\hline II & 101 & $47(54)$ & & $51(50)$ & & $51(50)$ & & $49(52)$ & \\
\hline III & 139 & $78(61)$ & & $69(70)$ & & $62(77)$ & & $69(70)$ & \\
\hline IV & 33 & $15(18)$ & & $15(18)$ & & $19(14)$ & & $19(14)$ & \\
\hline \multicolumn{10}{|c|}{ Invasion depth } \\
\hline $\mathrm{T} 1$ & 15 & $9(6)$ & $0.023 *$ & $12(3)$ & $0.049 *$ & $11(4)$ & 0.090 & $9(6)$ & 0.216 \\
\hline $\mathrm{T} 2$ & 63 & $21(42)$ & & $33(30)$ & & $37(26)$ & & $28(35)$ & \\
\hline T3 & 151 & $84(67)$ & & $77(74)$ & & $69(82)$ & & $70(81)$ & \\
\hline $\mathrm{T} 4$ & 66 & $44(42)$ & & $36(50)$ & & $41(45)$ & & $50(36)$ & \\
\hline Lymph & & & & & & & & & \\
\hline \multicolumn{10}{|c|}{$\operatorname{metastasis}(\mathrm{N})$} \\
\hline No & 99 & $46(53)$ & 0.348 & $49(50)$ & 0.828 & $52(47)$ & 0.864 & $50(49)$ & 0.206 \\
\hline $\mathrm{N} 1$ & 83 & $48(35)$ & & $40(43)$ & & $43(40)$ & & $44(39)$ & \\
\hline $\mathrm{N} 2$ & 69 & $31(38)$ & & $38(31)$ & & $33(36)$ & & $27(42)$ & \\
\hline N3 & 64 & $33(31)$ & & $31(33)$ & & $30(34)$ & & $36(28)$ & \\
\hline \multicolumn{10}{|c|}{ Distant metastasis } \\
\hline No & 295 & $149(146)$ & 0.652 & $149(146)$ & 0.652 & $145(150)$ & 0.248 & $144(151)$ & 0.174 \\
\hline Yes & 20 & $9(11)$ & & $9(11)$ & & $13(7)$ & & $13(7)$ & \\
\hline \multicolumn{10}{|c|}{ Differentiation } \\
\hline $\mathrm{G} 1$ & 7 & $3(4)$ & 0.293 & $4(3)$ & 0.906 & $3(4)$ & 0.758 & $3(4)$ & $0.030 *$ \\
\hline $\mathrm{G} 2$ & 108 & $48(60)$ & & $55(53)$ & & $57(51)$ & & $43(65)$ & \\
\hline G3 & 200 & 107(93) & & $99(101)$ & & $98(102)$ & & $111(89)$ & \\
\hline
\end{tabular}

$2 * \mathrm{p}<0.05$

3 


\section{Table 3 (on next page)}

Table.3 Ongoing clinical trials evaluating decitabinedrug in solid tumors

Table.3 Ongoing clinical trials evaluating decitabine drug in solid tumors 
1 Table.3 Ongoing clinical trials evaluating decitabine drug in solid tumors

\begin{tabular}{|c|c|c|c|c|c|}
\hline Study number & Phase & Drug & disease & Status & Completion date \\
\hline NCT03875287 & $\mathrm{I}$ & $\begin{array}{l}\text { Decitabine } \\
\text { Cedazuridine }\end{array}$ & Solid Tumors & Recruiting & August 2021 \\
\hline NCT04049344 & II & $\begin{array}{l}\text { Combination of decitabine with } \\
\text { oxaliplatin }\end{array}$ & $\begin{array}{l}\text { Relapsed/Metastatic Renal Cell } \\
\text { Carcinoma }\end{array}$ & Recruiting & December, 2021 \\
\hline NCT03445858 & I & $\begin{array}{l}\text { Pembrolizumab } \\
\text { CombinedWith Decitabine }\end{array}$ & $\begin{array}{l}\text { Young Adult Patients With Relapsed } \\
\text { and Refractory Solid Tumors or } \\
\text { Lymphoma }\end{array}$ & Recruiting & January 12,2025 \\
\hline NCT02959164 & $\mathrm{Ib}$ & Decitabine and Gemcitabine & $\begin{array}{l}\text { Refractory Pancreatic } \\
\text { Adenocarcinoma and Advanced Soft } \\
\text { Tissue or Bone Sarcomas }\end{array}$ & $\begin{array}{l}\text { Active, } \\
\text { not recruiting }\end{array}$ & June, 2021 \\
\hline
\end{tabular}

2 